\title{
Distribución de LA DIATOMEa INVASORA DIDYMOSPHENIA GEMINATA (BACILLARIOPHYCEAE) EN CUERPOS DE AGUA patagónicos de Argentina
}

\author{
Distribution OF THE INVASIVE DiATOM DiDYMOSPHENIA GEMINATA \\ (BACILLARIOPHYCEAE) IN PATAgonIAN WATER BODIES OF ARgENTINA
}

\begin{abstract}
Anabel A. Lamaro ${ }^{1,2 *}\left(\mathbb{D}\right.$, Juliana Pisonero ${ }^{1}$, Noelia Uyua ${ }^{3,4}(\mathbb{D})$, Viviana Sastre ${ }^{4}$, Norma Santinelli4 ${ }^{4}$, Julieta Muñiz Saavedra ${ }^{5,6}$ (D) S Silvia E. Sala ${ }^{1}$ (D)
\end{abstract}

\begin{abstract}
1. División Ficología, Facultad de Ciencias Naturales y Museo UNLP. La Plata. Buenos Aires.

2. Comisión Nacional de Actividades Espaciales. CABA.

3. Consejo Nacional de Investigaciones Científicas y Técnicas.

4. Instituto de Investigación de Hidrobiología, Facultad de Ciencias Naturales y Ciencias de la Salud, Sede Trelew, UNPSJB. Chubut.

5. Dirección General de Biología Acuática, Secretaría de Ambiente y Desarrollo Sostenible. Neuquén.

6. Asentamiento Universitario San Martín de los Andes. UNCOMA. Neuquén.
\end{abstract}

*analamaro@fcnym.unlp.edu.ar

\section{Citar este artículo}

LAMARO, A. A., J. PISONERO, N. UYUA, V. SASTRE, N. SANTINELLI, J. MUÑIZ SAAVEDRA \& S. E. SALA. 2019. Distribución de la diatomea invasora Didymosphenia geminata (Bacillariophyceae) en cuerpos de agua patagónicos de Argentina. Bol. Soc. Argent. Bot. 54: 169-183.

DOI: http://dx.doi. org/10.31055/1851.2372.v54. n2.24362

Recibido: 17 Agosto 2018

Aceptado: 24 Enero 2019

Publicado: 30 Junio 2019

Editora: Eugenia Alicia Sar

\section{SUMMARY}

Background and aims: Didymosphenia geminata is a freshwater diatom that forms dense soggy carpet-like layers lining the bottom of aquatic environments. In Argentina the Secretaría de Ambiente y Desarrollo Sustentable de la Nación declared it exotic invader species. In the last 9 years it has colonized rivers from Patagonia and its distribution in the region has considerably widen. The aim of this paper is to analyze current distribution of this species invasion in Argentinean Patagonia through the use of Geographic Information Systems (GIS).

M\&M: The analyzed data were obtained by our team in Neuquén and Chubut provinces and also from technical reports published by universities or governmental institutions in websites. A database at regional scale from Neuquén to Tierra del Fuego was built considering presence-absence data, geographic position and dates and in some cases environmental data ( $\mathrm{pH}$, conductivity, etc.)

Results: The results were incorporated to a GIS using Q-Gis 2.14 an open source and free software that allowed us to generate distribution maps at national and provincial level.

Conclusions: The available data show that $D$. geminata has widen its distribution since 2010 to present from Chubut province to the north reaching Neuquén province and to the south up to Tierra del Fuego province.

\section{KEY WORDS}

Didymosphenia geminata, GIS, Patagonia, Argentina, distribution.

\section{RESUMEN}

Introducción y objetivos: Didymosphenia geminata, es una diatomea que fue declarada "Especie Exótica Invasora" en Argentina por la Secretaría de Ambiente y Desarrollo Sustentable de la Nación. Esta especie coloniza rocas y puede formar grandes matas mucilaginosas que cubren el lecho de los ríos, ocasionando un considerable impacto estético y provocando serias alteraciones en los ecosistemas. En los últimos 9 años la especie ha colonizado ríos andino-patagónicos y ha aumentado considerablemente su distribución en la región. El objetivo general de este trabajo es analizar la distribución actual de esta especie en la Patagonia argentina mediante el uso de Sistemas de Información Geográfica (SIG).

M\&M: Los datos provienen de resultados obtenidos por el grupo de trabajo en las provincias del Neuquén y del Chubut e información abierta y libre disponible en la web. Se generó una base de datos a escala regional abarcando desde Neuquén a Tierra del Fuego.

Resultados: Los resultados fueron incorporados a un SIG utilizando un software libre y abierto Q-GIS 2.14 lo que permitió la generación de mapas de distribución a nivel provincial y nacional.

Conclusiones: Los datos disponibles hasta marzo de 2018 muestran que $D$. geminata ha expandido su distribución entre 2010 y 2018 desde la provincia del Chubut hacia el norte alcanzando la provincia de Neuquén y hacia el sur llegando a Tierra del Fuego.

Palabras clave

Didymosphenia geminata, SIG, Patagonia, Argentina, distribución. 


\section{INTRODUCCIÓN}

Didymosphenia geminata (Lyngbye) Schmidt, también conocida como "didymo" o "moco de roca", es una diatomea (Bacillariophyceae) bentónica de agua dulce. La especie ha sido históricamente considerada característica de cuerpos de agua oligotróficos y fríos de regiones circumboreales de Europa, Asia y América del Norte (Blanco \& Ector, 2009; Whitton et al., 2009). Los registros más antiguos de floraciones masivas tienen más de 100 años en ríos de Escandinavia (Lindstrøm \& Skulberg, 2008) mientras que los registros más antiguos de floraciones en los EE.UU. son de hace 20 años (Bothwell \& Spaulding, 2008). A partir de entonces, investigadores del Hemisferio Norte han documentado la presencia de grandes floraciones en Canadá, Estados Unidos, Islandia, Polonia, Italia y España (Bothwell et al., 2014; Gillis \& Chalifour, 2010; Taylor \& Bothwell, 2014). Sin embargo, estos eventos no fueron descriptos como invasiones debido a que $D$. geminata ya estaba presente en esos países (Kilroy \& Unwin, 2011). En la última década ha sido reportada como una especie invasora agresiva en ríos oligotróficos de todo el mundo (Blanco \& Bécares Mantecón, 2009; Blanco \& Ector, 2009; Kilroy \& Unwin, 2011). En el Hemisferio Sur, las primeras grandes proliferaciones se reportaron en Nueva Zelanda a partir del año 2004 (Kilroy, 2004). Kilroy et al. (2007) y Kilroy (2008) señalaron que la especie habría sido introducida en esta región por el hombre, principalmente por pescadores deportivos de salmónidos que habitualmente se trasladan alrededor del mundo. La hipótesis de una invasión reciente ha sido corroborada por Kilroy \& Novis (2018) en base al análisis de datos ambientales históricos y a estudios moleculares. Sin embargo, en Nueva Zelanda hay al menos un reporte en una lista de especies de algas de agua dulce realizado por Chapman et al. (1957) en el que no se presentan ilustraciones ni se especifica número de colección de la muestra analizada. Además, Whitton et al. (2009) señalaron que la especie fue reportada en Australia por Day et al. (1995) como Gomphonema geminata en un listado de algas. Aunque estos son estudios florísticos generales, no hay razones para cuestionarlos más que a cualquier otro registro en la literatura clásica (Whitton et al., 2009).

McNyset \& Julius (2006) propusieron un mapa de distribución global de $D$. geminata basado en modelos de nicho ecológico donde se muestran los sitios en todos los continentes, a excepción de Antártida, con riesgo de ser invadidos por la especie. Ríos del Hemisferio Sur, en especial de la zona patagónica de Argentina y Chile, fueron identificados como vulnerables a nuevas introducciones e invasiones. Frente a esta alerta, se realizaron muestreos prospectivos en Chile en 2010. La especie fue hallada en el río Futaleufú perteneciente a una cuenca compartida entre Chile y Argentina (Reid et al., 2012; Sastre et al., 2013). En solo un año se confirmó su presencia en 20 ríos distribuidos a lo largo de $800 \mathrm{~km}$ en Chile (Reid et al., 2012). En Argentina, luego de su hallazgo en la provincia del Chubut (Sastre et al., 2010 a, b), fue hallada en Neuquén (Moreno et al., 2011), Río Negro (Beamud et al., 2013) y Tierra del Fuego (Sala et al., 2013).

Si bien las pautas de manejo de la especie están focalizadas en las actividades antrópicas como turismo y pesca, recientemente se ha mencionado como posible causa de la formación de estas proliferaciones masivas a la influencia a gran escala de la intervención humana en los procesos climáticos, atmosféricos y edáficos (Bothwell et al., 2014). Además, se ha demostrado en Chile que el visón americano (una especie introducida e invasora) puede actuar también como agente de dispersión (Bus Leone et al., 2014). Es importante señalar que en Argentina el visón también ha colonizado rápidamente ambientes patagónicos de sur a norte alcanzando en la actualidad a la provincia del Neuquén (comunicación personal Guardas ambientales de Lonco Luan). Independientemente de si la especie es nativa o introducida, está demostrado que coloniza rápidamente sustratos rocosos, pudiendo producir proliferaciones masivas que cubren grandes extensiones (Spaulding \& Elwell, 2007). Esta plaga provoca alteraciones físicas y estructurales de los ecosistemas acuáticos con el consiguiente perjuicio sobre la fauna de macroinvertebrados y peces (Larned et al., 2007; Whitton et al., 2009), el valor estético del paisaje $y$ el funcionamiento de obras hidráulicas, con impacto negativo desde el punto de vista ecológico, económico y social (Kilroy, 2005; Kilroy et al., 2005; Kilroy \& Dale, 2006). Los efectos adversos que causa en los ecosistemas fueron bien documentados (James \& Chipps, 2016; Kilroy, 2005; Kilroy et al., 2005; Kilroy \& Dale, 2006; Larned et al., 2007; 


\section{A. A. Lamaro et al. - Didymosphenia geminata en Argentina}

Sanmiguel et al., 2016; Whitton et al., 2009) por lo que su aparición en los ríos patagónicos alertó a la comunidad científica.

Contrariamente a lo que se pensaba hasta hace poco tiempo en relación a su distribución exclusiva en el Hemisferio Norte (Spaulding \& Elwell, 2007), la especie estaba presente en América del Sur, específicamente en Chile, al menos desde la década del 60 (Asprey et al., 1964; Rivera \& Gebauer, 1989). Sin embargo, de acuerdo a lo documentado en Vouilloud (2003) y publicaciones posteriores, en Argentina la especie no fue reportada hasta 2010. Considerando que la información básica en Argentina es escasa en relación a la extensión y variabilidad geográfica del territorio, es posible que al igual que en Chile, esta especie hubiera estado presente en pequeñas poblaciones. Independientemente de si se trata de una especie nativa o exótica, en la actualidad $D$. geminata está colonizando rápidamente nuevas áreas en la Patagonia. La Secretaría de Ambiente y Desarrollo Sustentable de la Nación la declaró "Especie Exótica Invasora" en Argentina (Res. 991/12) y estableció un grupo de trabajo intersectorial, el Comité Técnico Regional (CTR) conformado por organismos provinciales, municipales y privados. Este comité acuerda las acciones tendientes a la implementación de una estrategia para el control y la reducción de los riesgos de expansión del alga en el territorio nacional. Además, se implementaron programas de vigilancia, seguimiento y control en las provincias del Neuquén (Abelli Bonardi et al, 2012), Chubut, Santa Cruz y Tierra del Fuego, llevados a cabo por instituciones provinciales y avalados por el CTR y el Consejo Federal del Medio Ambiente.

Para la resolución de problemas ambientales similares a los ocasionados por D. geminata, en las últimas décadas se han incorporado nuevas herramientas tecnológicas de análisis. La necesidad de ampliar y aplicar el conocimiento científico disponible así como de integrar el conocimiento proveniente de otras disciplinas, hizo indispensable recurrir a modalidades de análisis que permitan el manejo de información con base territorial (Moreira-Muñoz, 1996). Los Sistemas de Información Geográfica (SIG) constituyen una herramienta que permite manejar información muy variada y compleja proveniente de fuentes diversas, facilitando el análisis simultáneo de varias dimensiones de un mismo problema (Burrough, 1986). Desde su aparición a fines de los '80, han sido de suma utilidad en estudios sobre conservación de la biodiversidad, análisis de especies como indicadores ambientales, estudios de impacto ambiental y de distribución de especies (Fitzhugh, 2005; Giansante \& Pelini, 2007; Haslett, 1990; Hijmans et al., 2001; Jones et al., 1997; Ramachandra \& Ahalya, 2001; Salem, 2003; Shultz, 2000, entre otros). En cuanto a estudios específicos relativos a distribución de diatomeas, pueden citarse trabajos relacionados a índices de calidad de agua (Behrendt \& Opitz, 2001; Naumoski et al., 2011), distribución de especies (Potapova \& Charles, 2002; Revenga et al., 2005) y estudios sobre especies invasoras (Blanco \& Ector, 2009; Kumar et al., 2009).

El objetivo general de este trabajo es analizar la distribución actual de D. geminata en Argentina hasta el otoño de 2018 mediante el uso de SIG.

\section{Materiales y MÉtodos}

Los datos relacionados con $D$. geminata fueron obtenidos de diversas fuentes (Tabla 1):

- Información obtenida por el grupo de trabajo, los datos se obtuvieron a partir de muestreos realizados siguiendo los protocolos nacionales (Casco \& Sala, 2012) e internacionales (Duncan et al., 2007). En cada sitio se estableció un tramo de río de 50-100 m de longitud (en función de la accesibilidad) a lo largo del cual se colectaron los diferentes sustratos disponibles. Los sustratos duros fueron cepillados con cepillo de dientes y las macrófitas y macroalgas fueron exprimidas. En todos los sitios además se tomaron muestras con red de plancton de $30 \mu \mathrm{m}$ de apertura de malla. Las muestras se fijaron in situ con formol al $4 \%$. El análisis de las muestras se realizó con microscopio óptico. Los registros negativos se establecieron cuando la especie no apareció en 10 preparados de las muestras de fitoplancton y perifiton. En los ambientes cordilleranos de la provincia del Chubut, la metodología utilizada fue descripta en Sastre et al. (2013) y Uyua et al. (2016).

- Información disponible en la web en forma de informes técnicos llevados a cabo por universidades e instituciones estatales encargadas del monitoreo de la especie en las provincias del Neuquén, Río 
Bol. Soc. Argent. Bot. 54 (2) 2019

Tabla 1. Fuentes de información de registros positivos y negativos de D. geminata incluidas en el análisis.

\begin{tabular}{|c|c|c|c|c|c|}
\hline Provincia & Publicación / Informe & Institución & Fuente de información & $\begin{array}{l}\text { Tipo de } \\
\text { Muestra }\end{array}$ & $\begin{array}{l}\text { Frecuencia } \\
\text { de muestreo }\end{array}$ \\
\hline \multirow[t]{4}{*}{ Neuquén } & Informes Nro. 1 al 10 & $\begin{array}{l}\text { Centro de Ecología } \\
\text { Aplicada de } \\
\text { Neuquén (CEAN) }\end{array}$ & $\begin{array}{l}\text { http://www.cean.gob.ar/ } \\
\text { alerta-didymo/. Último } \\
\text { acceso: marzo } 2018\end{array}$ & $\begin{array}{l}\text { fitoplancton } \\
\text { y perifiton }\end{array}$ & $\begin{array}{l}\text { estacional } \\
2011 \text { a } 2016\end{array}$ \\
\hline & $\begin{array}{l}\text { Informe de presencia } \\
\text { de Didymosphenia } \\
\text { geminata. Provincia } \\
\text { del Neuquén- } \\
\text { Marzo de } 2015\end{array}$ & $\begin{array}{l}\text { Dirección General } \\
\text { de Biología } \\
\text { Acuática, San } \\
\text { Martín de los Andes }\end{array}$ & $\begin{array}{l}\text { Muñiz Saavedra, com. } \\
\text { pers. marzo } 2015\end{array}$ & $\begin{array}{l}\text { fitoplancton } \\
\text { y perifiton }\end{array}$ & estacional 2014 \\
\hline & Beamud et al., 2016 & & & perifiton & $\begin{array}{l}\text { diciembre } \\
\text { de } 2013\end{array}$ \\
\hline & $\begin{array}{l}\text { Muestreo del grupo } \\
\text { de trabajo en el } \\
\text { marco de proyecto } \\
\text { de Extensión de } \\
\text { Voluntario Universitario. } \\
\text { Convocatorias } \\
2015-2018\end{array}$ & & datos propios & $\begin{array}{l}\text { fitoplancton } \\
\text { y perifiton }\end{array}$ & $\begin{array}{l}\text { estacional } \\
2017-2018\end{array}$ \\
\hline \multirow[t]{2}{*}{ Río Negro } & $\begin{array}{l}\text { Beamud et al., } \\
2013,2016\end{array}$ & & & perifiton & $\begin{array}{l}\text { enero de } 2013 \\
\text { y } 2014\end{array}$ \\
\hline & $\begin{array}{l}\text { Información publicada } \\
\text { en la web }\end{array}$ & $\begin{array}{l}\text { Gobierno de } \\
\text { Río Negro }\end{array}$ & $\begin{array}{l}\text { http://www.ambiente. } \\
\text { rionegro.gov.ar/index. } \\
\text { php?contID=30034. } \\
\text { Último acceso: } \\
\text { enero } 2017\end{array}$ & perifiton & abril de 2016 \\
\hline \multirow[t]{4}{*}{ Chubut } & Beamud et al, 2016 & & & perifiton & diciembre de 2013 \\
\hline & Sastre et al, 2013 & & datos propios & $\begin{array}{l}\text { fitoplancton } \\
\text { y perifiton }\end{array}$ & $\begin{array}{l}\text { mensual } 2010 \text { a } \\
2012 ; \text { estacional } \\
2013 \text { a } 2015\end{array}$ \\
\hline & $\begin{array}{l}\text { Mapa monitoreo } \\
\text { histórico 2010-2016 }\end{array}$ & $\begin{array}{l}\text { Ministerio de } \\
\text { Ambiente y Control } \\
\text { del Desarrollo } \\
\text { Sustentable } \\
\text { de la provincia } \\
\text { del Chubut }\end{array}$ & $\begin{array}{l}\text { http://www.chubut.gov. } \\
\text { ar/portal/wp-organismos/ } \\
\text { ambiente/plan- } \\
\text { provincial-didymo/. } \\
\text { Último acceso: } \\
\text { enero } 2017\end{array}$ & $\begin{array}{l}\text { fitoplancton } \\
\text { y perifiton }\end{array}$ & $\begin{array}{l}\text { estacional } \\
2010 \text { a } 2016\end{array}$ \\
\hline & $\begin{array}{l}\text { Informes de campaña } \\
(2015-2017)\end{array}$ & $\begin{array}{l}\text { Facultad de } \\
\text { Ciencias Naturales } \\
\text { y Ciencias de la } \\
\text { Salud (FCNyCS), } \\
\text { UNPSJB. Sedes } \\
\text { Trelew/Esquel } \\
\text { y Ministerio de } \\
\text { Ambiente y Control } \\
\text { del Desarrollo } \\
\text { Sustentable } \\
\text { del Chubut }\end{array}$ & $\begin{array}{l}\text { http://www.chubut.gov. } \\
\text { ar/portal/wp-organismos/ } \\
\text { ambiente/plan- } \\
\text { provincial-didymo/. } \\
\text { Último acceso: } \\
\text { marzo } 2018\end{array}$ & $\begin{array}{l}\text { fitoplancton } \\
\text { y perifiton }\end{array}$ & $\begin{array}{l}\text { estacional } \\
2016-2017\end{array}$ \\
\hline \multirow[t]{3}{*}{$\begin{array}{l}\text { Santa } \\
\text { Cruz }\end{array}$} & Uyua, 2017 & & datos propios & $\begin{array}{l}\text { fitoplancton } \\
\text { y perifiton }\end{array}$ & marzo de 2015 \\
\hline & Braccalenti, 2016 & & & perifiton & $\begin{array}{l}\text { octubre, } \\
\text { diciembre } 2014 \text {, } \\
\text { marzo } 2015\end{array}$ \\
\hline & $\begin{array}{l}\text { Muestreo del grupo } \\
\text { de trabajo }\end{array}$ & & datos propios & perifiton & enero de 2017 \\
\hline
\end{tabular}




\section{A. A. Lamaro et al. - Didymosphenia geminata en Argentina}

Tabla 1. Continuación.

\begin{tabular}{|c|c|c|c|c|c|}
\hline Provincia & Publicación / Informe & Institución & Fuente de información & $\begin{array}{l}\text { Tipo de } \\
\text { Muestra }\end{array}$ & $\begin{array}{l}\text { Frecuencia } \\
\text { de muestreo }\end{array}$ \\
\hline \multirow[t]{2}{*}{$\begin{array}{l}\text { Tierra del } \\
\text { Fuego }\end{array}$} & $\begin{array}{l}\text { Informe de monitoreo } \\
\text { Año } 2013\end{array}$ & $\begin{array}{l}\text { Secretaría } \\
\text { de Desarrollo } \\
\text { Sustentable y } \\
\text { Ambiente de } \\
\text { la provincia de } \\
\text { Tierra del Fuego }\end{array}$ & $\begin{array}{l}\text { http://recursoshidricos. } \\
\text { tierradelfuego.gov.ar. } \\
\text { Último acceso: } \\
\text { enero } 2017\end{array}$ & perifiton & abril de 2013 \\
\hline & Sala et al., 2013 & & datos propios & perifiton & febrero de 2013 \\
\hline
\end{tabular}

Negro, Chubut, Santa Cruz y Tierra del Fuego. En todos los casos, se utilizó información disponible de manera abierta y libre, que fue corroborada mediante la consulta con investigadores involucrados en el tema. Esta información está respaldada por contar con una metodología de muestreo y análisis detallada.

La información obtenida en diversos formatos, fue estandarizada en una planilla de base de datos. Los datos mínimos requeridos para el ingreso a la base fueron: registro positivo o negativo de la especie, fecha de muestreo, coordenadas geográficas del sitio y fuente de información. En algunos pocos casos pudo obtenerse información ambiental ( $\mathrm{pH}$, conductividad, temperatura, etc.), que fue incorporada a la base de datos como datos complementarios junto con otros datos anexos (cuenca, características del sitio de muestreo, etc.).

El procesamiento y análisis de datos se realizó utilizando planillas de cálculo, conversores de coordenadas, conversores de formatos: Global Mapper y Google Earth. El programa de SIG utilizado es el Q-Gis Versión 2.14, de uso libre y código abierto (http://www.qgis.org/es/site/).

Como base cartográfica y temática para el SIG se buscó y seleccionó información oficial de Argentina. Los datos geográficos obtenidos del Instituto Geográfico Nacional (IGN) fueron: cursos y cuerpos de agua (de ambas coberturas se seleccionaron sólo los permanentes) y límites administrativos provinciales y nacionales. Esta información proviene, en su mayoría, del conjunto de datos surgido del proyecto SIG 250, realizado por el IGN, con permanente actualización de la base de datos geográfica y adecuación a las diferentes normas y estándares. Otros datos geográficos específicos como: regiones ecológicas (o ecorregiones) y áreas protegidas fueron suministrados por la Administración de
Parques Nacionales (APN), y Sistema Federal de Áreas Protegidas (SIFAP) del Ministerio de Ambiente y Desarrollo Sustentable de la Nación.

Todos los datos fueron obtenidos mediante descarga directa de los archivos o mediante Web Services (WMS y WFS).

La metodología empleada en las diferentes etapas de procesamiento de la información se realizó siguiendo el esquema de la Fig. 1.

Todas las capas vectoriales originales, fueron editadas a fin de recortar solo el área de trabajo (Patagonia argentina) y unificar formatos y reproyectadas al sistema de referencia seleccionado: POSGAR 2007 Faja 2.

\section{Resultados}

Se obtuvieron un total de 836 registros (positivos y negativos) de D. geminata, desde septiembre de 2010 (primera aparición en Argentina) hasta abril de 2018. El número de estos registros así como los subtotales por provincia se detalla en la Tabla 2.

En las provincias del Neuquén, Río Negro, Santa Cruz y Tierra del Fuego los registros públicos (informes disponibles en la web o publicaciones científicas) son discontinuos en el tiempo. En la provincia del Chubut los muestreos fueron realizados sistemáticamente en primavera y otoño desde el primer registro de la especie, en muchos casos por el grupo de trabajo.

Un análisis preliminar temporal y espacial de la expansión de $D$. geminata se realizó en base a las capas de información generadas en el SIG.

Distribución de D. geminata en Neuquén y Rio Negro, 2011-2014 y 2016-2018

Estas provincias son analizadas en conjunto (181 registros) debido a que en la provincia de 
Bol. Soc. Argent. Bot. 54 (2) 2019

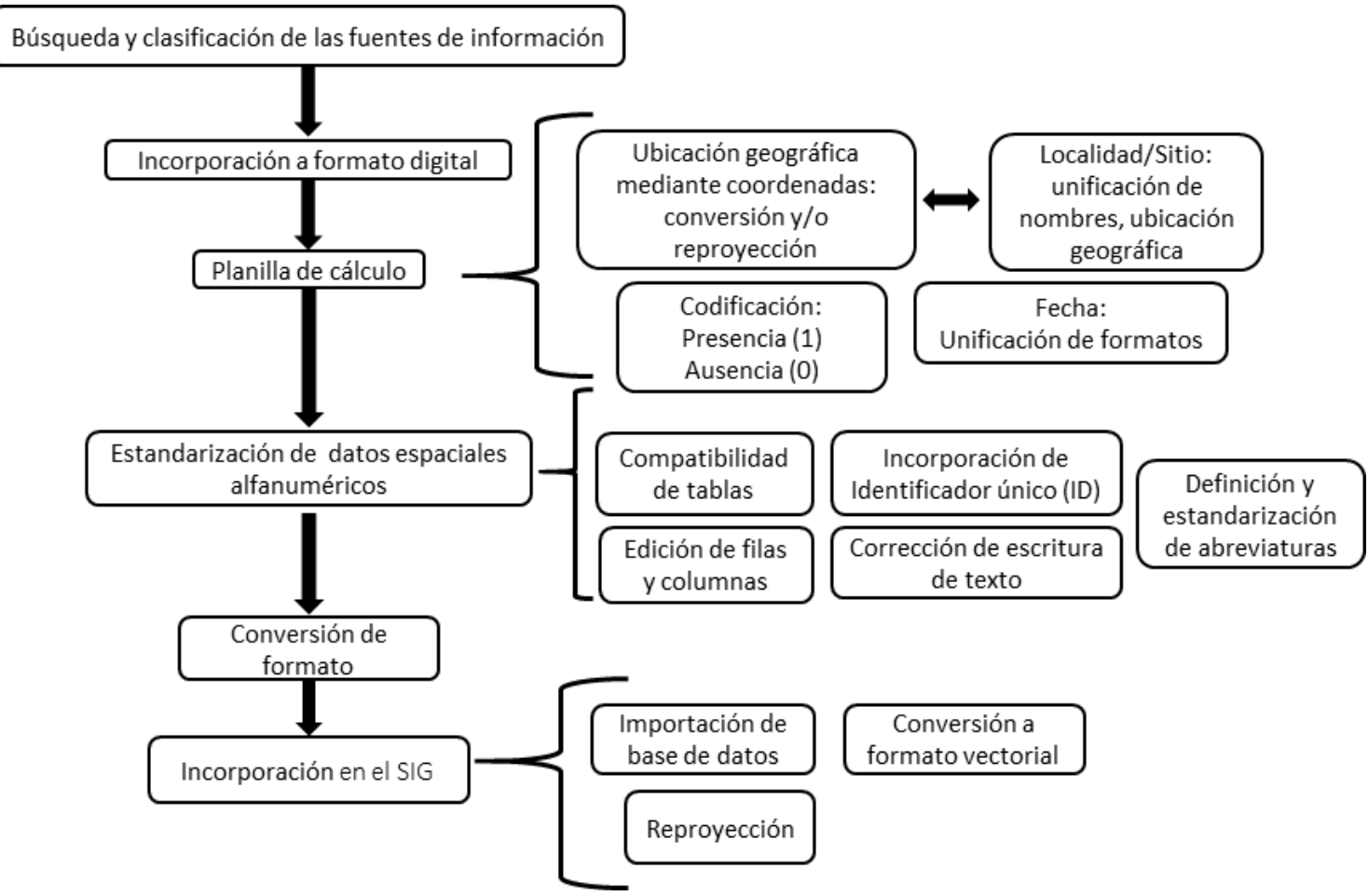

Fig. 1. Esquema con las etapas de procesamiento de la información.

Tabla 2. Registros de D. geminata por provincia y por año. Referencias: s/d: sin datos.

\begin{tabular}{|c|c|c|c|c|c|c|c|c|c|c|}
\hline \multirow[t]{2}{*}{ Año } & \multicolumn{2}{|c|}{$\begin{array}{l}\text { Neuquén } \\
\text { Registros }\end{array}$} & \multicolumn{2}{|c|}{$\begin{array}{l}\text { Río Negro } \\
\text { Registros }\end{array}$} & \multicolumn{2}{|c|}{$\begin{array}{l}\text { Chubut } \\
\text { Registros }\end{array}$} & \multicolumn{2}{|c|}{$\begin{array}{l}\text { Santa Cruz } \\
\text { Registros }\end{array}$} & \multicolumn{2}{|c|}{$\begin{array}{l}\text { Tierra del Fuego } \\
\text { Registros }\end{array}$} \\
\hline & Positivos & Negativos & Positivos & Negativos & Positivos & Negativos & Positivos & Negativos & Positivos & Negativos \\
\hline 2010 & $\mathrm{~s} / \mathrm{d}$ & $\mathrm{s} / \mathrm{d}$ & $\mathrm{s} / \mathrm{d}$ & $s / d$ & 11 & 75 & $s / d$ & $\mathrm{~s} / \mathrm{d}$ & $s / d$ & $\mathrm{~s} / \mathrm{d}$ \\
\hline 2011 & 8 & 8 & $s / d$ & $s / d$ & 15 & 106 & $s / d$ & $s / d$ & $s / d$ & $s / d$ \\
\hline 2012 & 13 & 9 & $\mathrm{~s} / \mathrm{d}$ & $s / d$ & 30 & 89 & $s / d$ & $\mathrm{~s} / \mathrm{d}$ & $\mathrm{s} / \mathrm{d}$ & $s / d$ \\
\hline 2013 & 11 & 9 & 1 & $s / d$ & 28 & 25 & $s / d$ & $s / d$ & 10 & 41 \\
\hline 2014 & 17 & 54 & 1 & $s / d$ & 15 & 30 & 2 & 60 & $s / d$ & $s / d$ \\
\hline 2015 & $s / d$ & $s / d$ & $s / d$ & $s / d$ & 18 & 26 & 2 & 13 & $s / d$ & $\mathrm{~s} / \mathrm{d}$ \\
\hline 2016 & 2 & 2 & $\mathrm{~s} / \mathrm{d}$ & 3 & 11 & 36 & $\mathrm{~s} / \mathrm{d}$ & $\mathrm{s} / \mathrm{d}$ & $s / d$ & $\mathrm{~s} / \mathrm{d}$ \\
\hline 2017 & 10 & 18 & $s / d$ & $s / d$ & 0 & 6 & 2 & 4 & $s / d$ & $s / d$ \\
\hline 2018 & 9 & 6 & $s / d$ & $\mathrm{~s} / \mathrm{d}$ & $\mathrm{s} / \mathrm{d}$ & $\mathrm{s} / \mathrm{d}$ & $\mathrm{s} / \mathrm{d}$ & $s / d$ & $\mathrm{~s} / \mathrm{d}$ & $s / d$ \\
\hline $\begin{array}{c}\text { Subtotales } \\
\text { por } \\
\text { provincia }\end{array}$ & 70 & 106 & 2 & 3 & 128 & 393 & 6 & 77 & 10 & 41 \\
\hline $\begin{array}{l}\text { Totales por } \\
\text { provincia }\end{array}$ & & 76 & & 5 & & 21 & & 33 & & 51 \\
\hline
\end{tabular}




\section{A. A. Lamaro et al. - Didymosphenia geminata en Argentina}

Río Negro solo se contabilizaron 5 registros: 2 registros positivos en 2013 y 2014 y 3 registros negativos en 2017. Los datos analizados de la provincia del Neuquén (176 registros: 70 positivos y 106 negativos) provienen de muestreos llevados a cabo por instituciones provinciales y por el grupo de trabajo y abarcan el período 2011-2014 y 2016-2018. El 94\% de los registros positivos de ambas provincias se encontraron en la cuenca del río Limay.

Los primeros registros positivos se hallaron en 2011, en la provincia del Neuquén en los ríos Chimehuín y Collón-Curá, en el tramo paralelo a la Ruta Provincial $\mathrm{N}^{\circ} 49$, entre las Rutas Nacionales $\mathrm{N}^{\mathrm{o}} 40$ y 234 . Estos registros tuvieron continuidad hasta 2014, sumándose positivos hacia el norte y el sur sobre el río Collón-Curá.

En los años 2012 y 2013 se registró positivamente en el río Malleo, en su confluencia con el río Aluminé, en el mismo sitio muestreado el año anterior con resultado negativo.

También en el año 2013 se registró positivamente en cercanías de las nacientes del río Limay y a orillas del lago Nahuel Huapi en la provincia de Río Negro siendo ésta la primera información de estos sitios.

Durante el año 2014, en la provincia del Neuquén se realizó un muestreo extenso en 72 sitios que abarcó la zona cordillerana y la estepa, incluyendo los ríos Neuquén, Limay y Colorado. Se encontraron nuevos registros positivos en sitios monitoreados anteriormente con resultado negativo, en los ríos (mencionados de norte a sur): Agrio (sobre Ruta Provincial $\mathrm{N}^{\circ}$ 21 y Arroyo Huarenchenque), Aluminé (sobre la Ruta Provincial $\mathrm{N}^{0} 23$ hacia el sur de la desembocadura del río Pulmarí), Quillén (cerca de su desembocadura en el río Aluminé), Chachín (en cercanías al lago Nonthué), Caleufú (en sus nacientes -río Filo Hua Hum y Meliquina- y en su desembocadura -río Collón Curá-), Traful (en su naciente en el lago del mismo nombre) y en el arroyo Chapelco Chico (a la altura del km 2220 de la Ruta Nacional No 40).

En el año 2016, detectamos la presencia de D. geminata en el río Aluminé, en su naciente en el lago homónimo y en un arroyo cercano (China Muerta). Durante el 2017 se amplió el área de muestreo a otros puntos a lo largo del río Aluminé y afluentes y en cercanías de
Villa Pehuenia con registros positivos en: lago Aluminé, arroyos Lonco Luan y Rebolledo, ríos Litrán, Pulmari y Ruca Choroi. También se registró positivamente en el lago Norquinco. Los últimos muestreos, de abril de 2018, mostraron 9 registros positivos, 2 en sitios no muestreados anteriormente (arroyos Moquehue y Remeco) y uno en el arroyo Quillahué, en la misma locación muestreada con resultado negativo el año anterior.

Por otra parte, el gobierno de Río Negro realizó un muestreo en territorio del Área Natural Protegida río Azul - lago Escondido con resultado negativo. El mapa de distribución del período analizado se muestra en la Fig. 2.

\section{Distribución de D. geminata en Chubut, 2010 -} 2017

Los registros en la provincia del Chubut tienen continuidad temporal durante todo el período analizado y son los más numerosos, un total de 521 con 128 positivos y 393 negativos (Fig. 3). Los muestreos se llevaron a cabo por instituciones provinciales y por el grupo de trabajo.

En esta provincia es donde se registró $D$. geminata por primera vez para Argentina, en Agosto de 2010 en el río Futaleufú, cerca del límite con Chile (Sastre et al. 2010a, b; Sastre et $a l ., 2013)$. Durante ese año se tomaron muestras en 86 sitios a lo largo de los principales ríos cordilleranos de la provincia, registrándose positivamente en 11 sitios en el río Futaleufú en el límite con Chile.

En el año 2011 (121 sitios muestreados: 15 positivos y 106 negativos) la especie se encontró nuevamente en los mismos sitios del río Futaleufú del año anterior y se registró positivamente en el río Pampa (cerca de la llamada Aldea Las Pampas sobre la Ruta Provincial No 19), distante $100 \mathrm{~km}$ del anterior, en el mismo sitio donde en el año 2010 se había obtenido resultado negativo.

En 2012, hubo 119 registros (30 positivos y 89 negativos). D. geminata fue encontrada nuevamente en el río Futaleufú y en sitios cercanos anteriormente muestreados con resultado negativo, como el arroyo Baguilt y la Estación de Piscicultura. Adicionalmente, se registró positivamente también en los ríos Arrayanes y Rivadavia y arroyo Colehual, ubicados entre los 
Bol. Soc. Argent. Bot. 54 (2) 2019

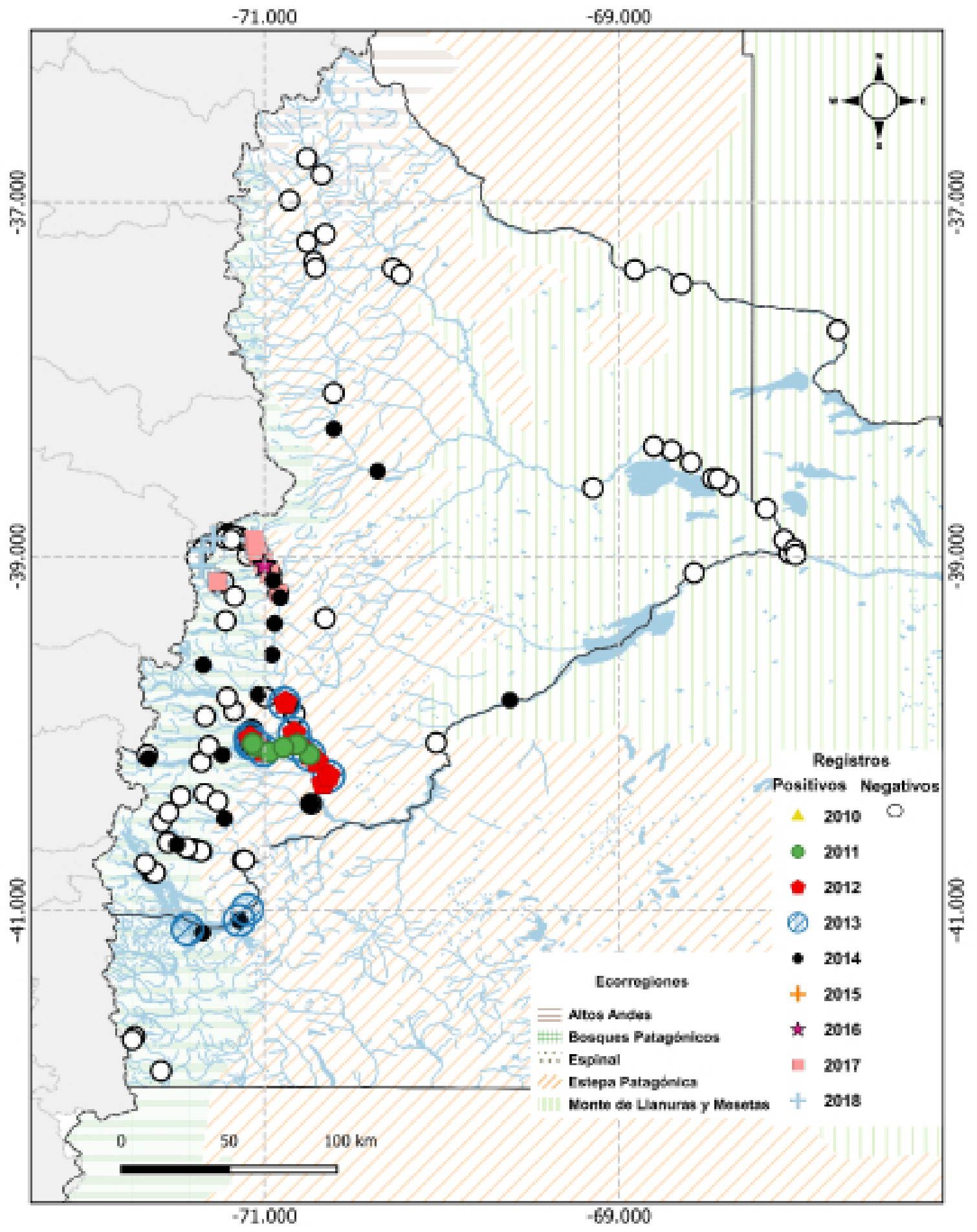

Fig. 2. Mapa de distribución de D. geminata en las provincias del Neuquén y Río Negro (2011-2014 y 20162018). 


\section{A. A. Lamaro et al. - Didymosphenia geminata en Argentina}

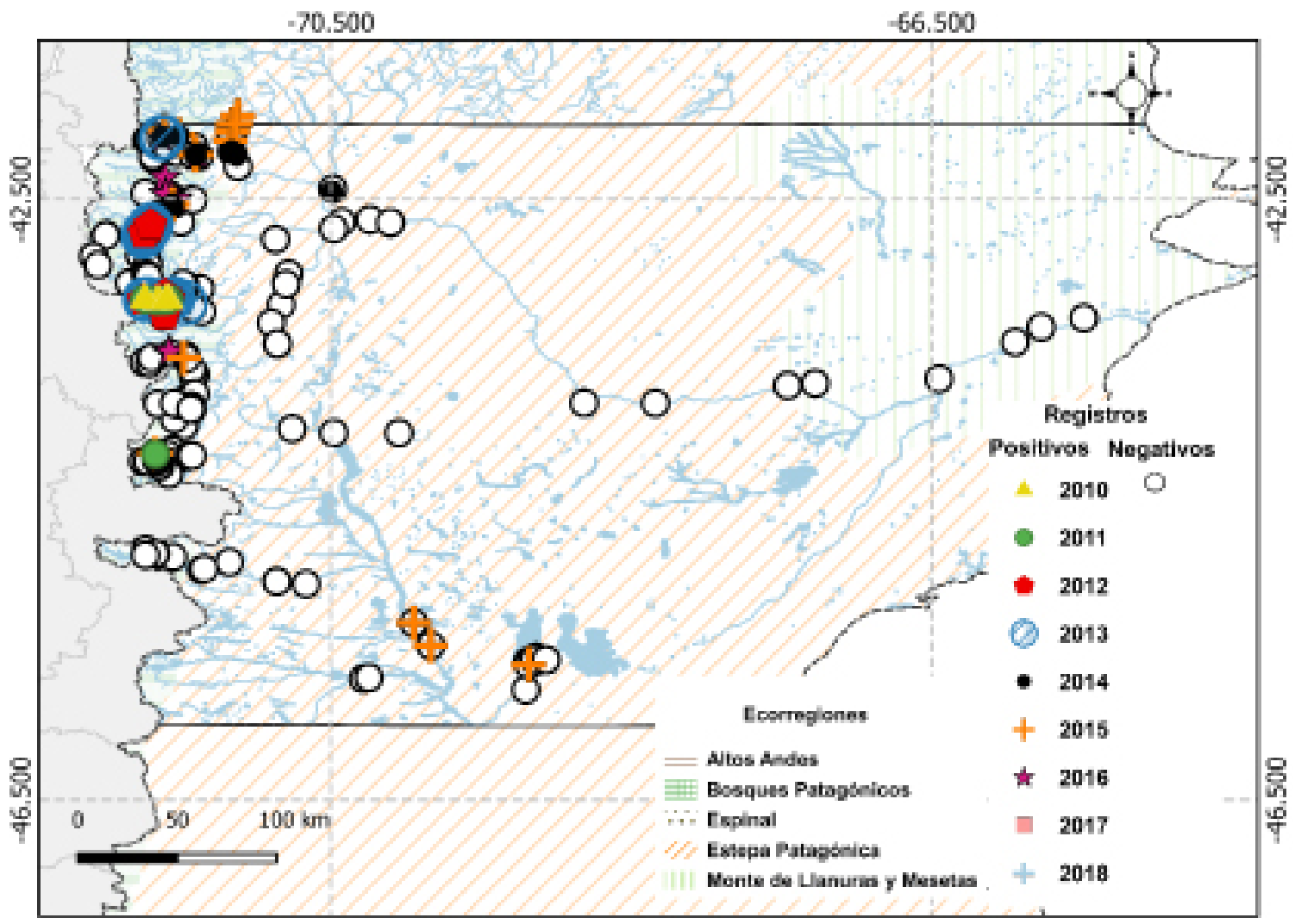

Fig. 3. Mapa de distribución de D. geminata en la provincia del Chubut (2010 a 2017).

lagos Rivadavia y Verde y distantes $50 \mathrm{~km}$ hacia el norte del río Futaleufú. Estos sitios habían sido muestreados en temporadas anteriores con resultado negativo.

En 2013 se muestrearon 53 sitios (con 28 registros positivos y 25 negativos), algunos de los registros positivos fueron en sitios con registros negativos previos, estos son: el río Azul (en su desembocadura en el lago Puelo) en el norte de la provincia y en los ríos Corintos (afluente del Futaleufú) y Nant y Fall (subsidiario del Corintos).

En 2014, hubo 15 registros positivos de un total de 45 muestreos. Los sitios con registros negativos en años anteriores fueron, en el sector norte de la provincia, en el río Epuyén (cerca del registro del año anterior en el río Azul) y en el río Chubut en dos sectores (sobre la Ruta Nacional $\mathrm{N}^{\circ} 40$ a 10 $\mathrm{km}$ al sur de la localidad de El Maitén y cerca de la confluencia con el río Chico hacia el oeste de la provincia). Luego, hacia el sur, se encontró en el río Carrileufú (cerca de la desembocadura en el lago Rivadavia) y en el río Pico (sitio cercano al registro positivo de 2010 en el río Pampa).

En 2015, hubo 18 registros positivos de un total de 44. Se encontró D. geminata en sitios sobre el río Chubut hacia el norte y hacia el sur de la localidad de El Maitén y en el río Senguer ubicado al sudoeste de la provincia. En 2016 hubo 11 positivos de un total de 47 . Estos ocurrieron en sitios previamente muestreados con registro negativo, como el arroyo Pedregoso (en cercanías del lago Cholila) y el arroyo Las Minas (cerca de su unión con el río Epuyén). En el mismo año se relevaron sitios a lo largo del río Chubut hasta casi su desembocadura con resultados negativos. En 2017 se muestrearon 6 puntos en cercanías del lago Musters, todos con resultado negativo.

Distribución de D. geminata en Santa Cruz, 20142015, 2017

De la provincia de Santa Cruz hay datos de 


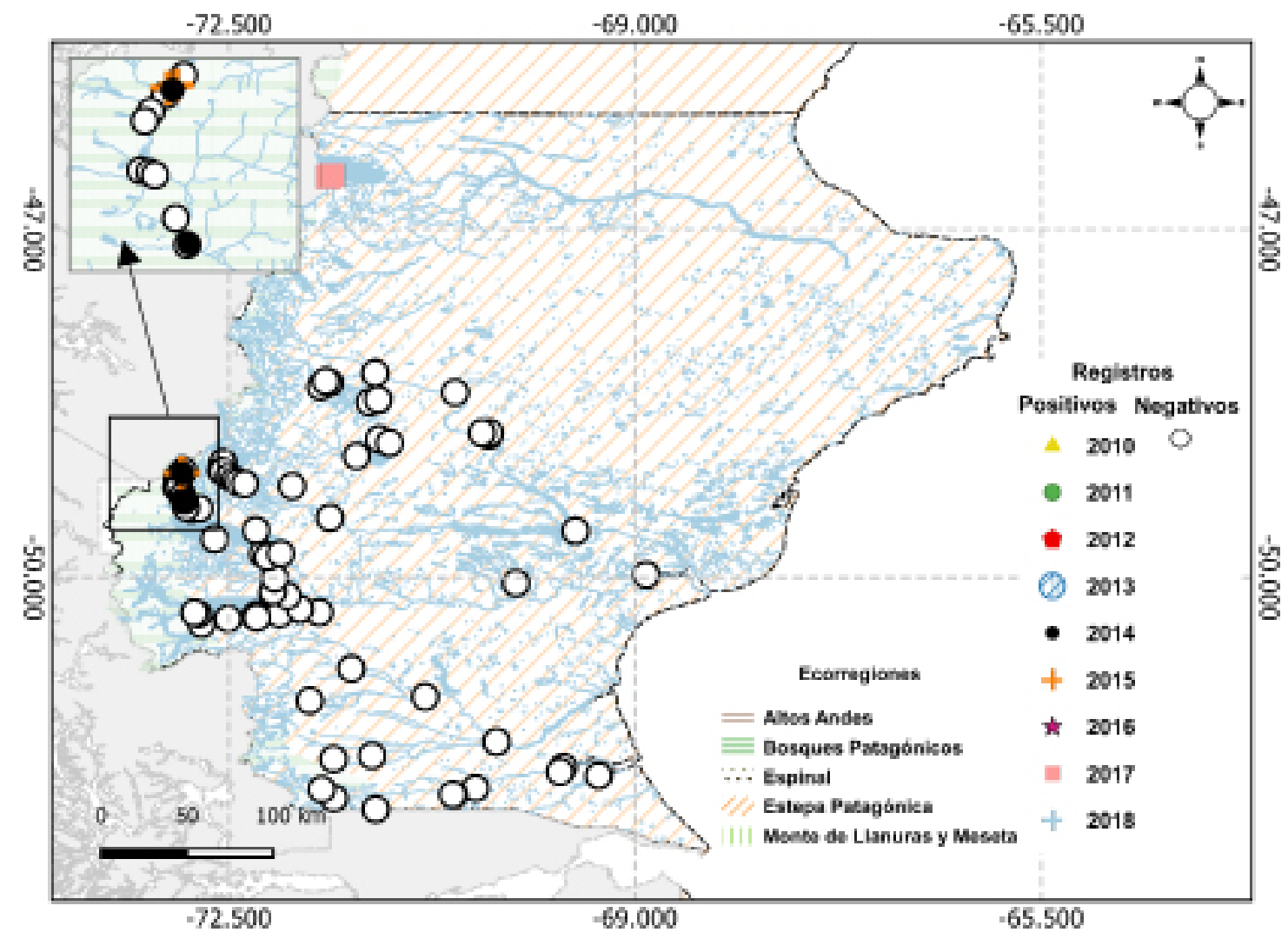

Fig. 4. Mapa de distribución de D. geminata en la provincia de Santa Cruz (2014-2015 y 2017).

los años 2014, 2015 y 2017 (Fig. 4). Del primer año mencionado se cuenta con información de 62 sitios relevados en un muestreo llevado a cabo por el Gobierno de Santa Cruz, de los cuales se registraron 2 positivos en el río de las Vueltas, uno en sus nacientes (sobre la Ruta Provincial $\mathrm{N}^{\circ} 23$, cerca de la laguna del Desierto) y el otro cercano al pueblo El Chaltén (distante aproximadamente $25 \mathrm{~km}$ del punto anterior). De los 15 sitios relevados en el 2015, también por el gobierno provincial, dieron positivo 2 sitios en el río de las Vueltas, cercano a las nacientes, al igual que el año anterior. Los positivos de 2014 y 2015, fueron corroborados por integrantes del grupo de trabajo. En el año 2017, colectamos muestras de 6 sitios (3 en cercanías de lago Argentino y los otros cerca del lago Buenos Aires) con 2 registros positivos, en el último lago mencionado y en el río Los Antiguos.
Distribución de D. geminata en Tierra del Fuego, 2013

El primer registro de D. geminata en Tierra del Fuego ocurrió en febrero de 2013 (Sala et al. 2013). Previamente a esa fecha, a diferencia de lo sucedido en otras provincias, los organismos provinciales y municipales de gestión no organizaron muestreos referidos a la presencia de la especie en la isla (Fig. 5). Durante el mismo año, la Secretaría de Desarrollo Sustentable y Ambiente de la provincia relevó cursos y cuerpos de agua en 51 sitios de la Isla Grande, con 10 registros positivos solo en el río Grande. Cabe destacar que este río nace en Chile y que los registros de D. geminata en ese país son de Marzo de 2013.

En la Fig. 6 y a modo de resumen se muestra la distribución acumulada año a año de $D$. geminata en toda la región patagónica. 
A. A. Lamaro et al. - Didymosphenia geminata en Argentina

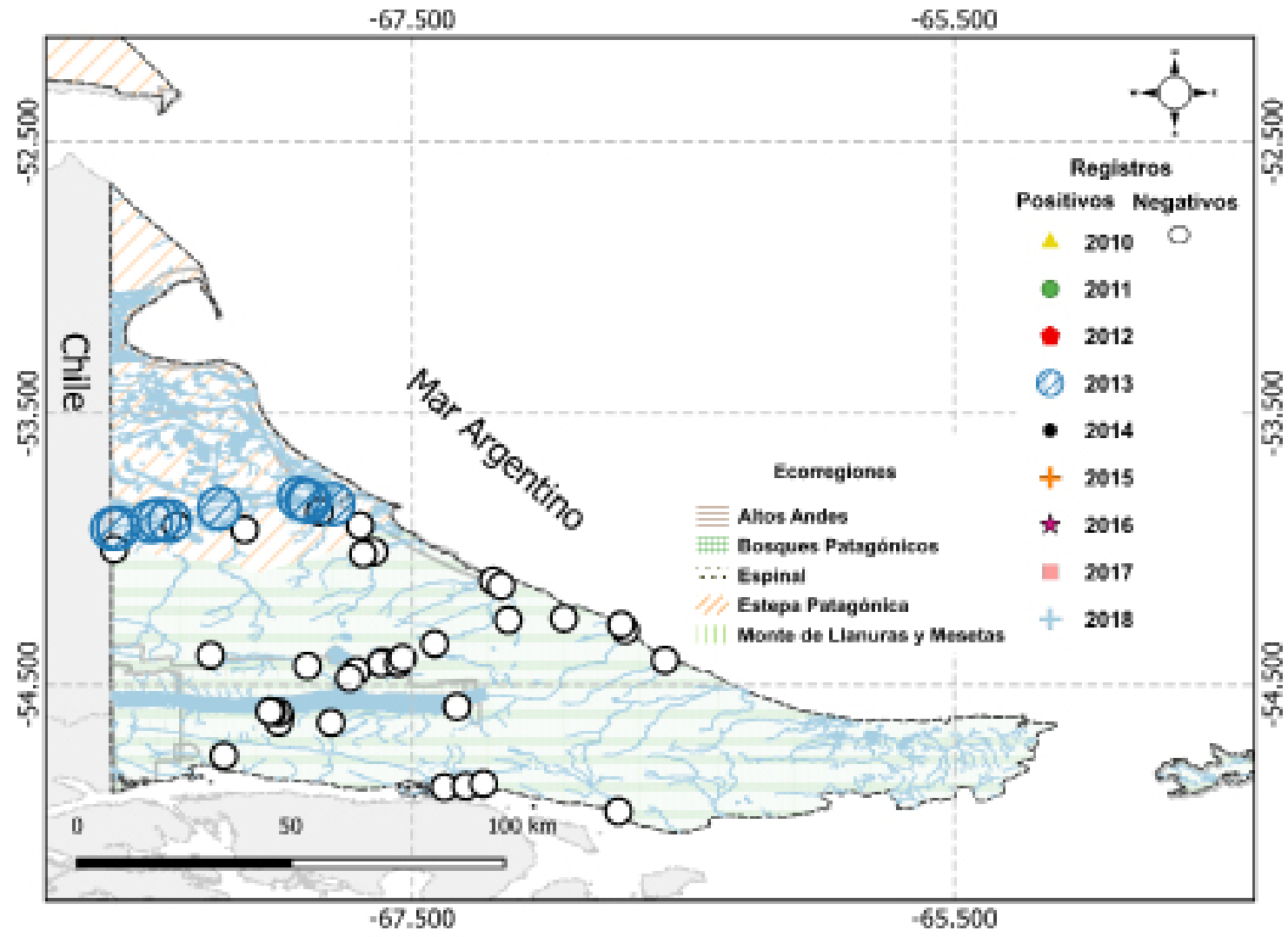

Fig. 5. Mapa de distribución de D. geminata en la provincia de Tierra del Fuego (2013).

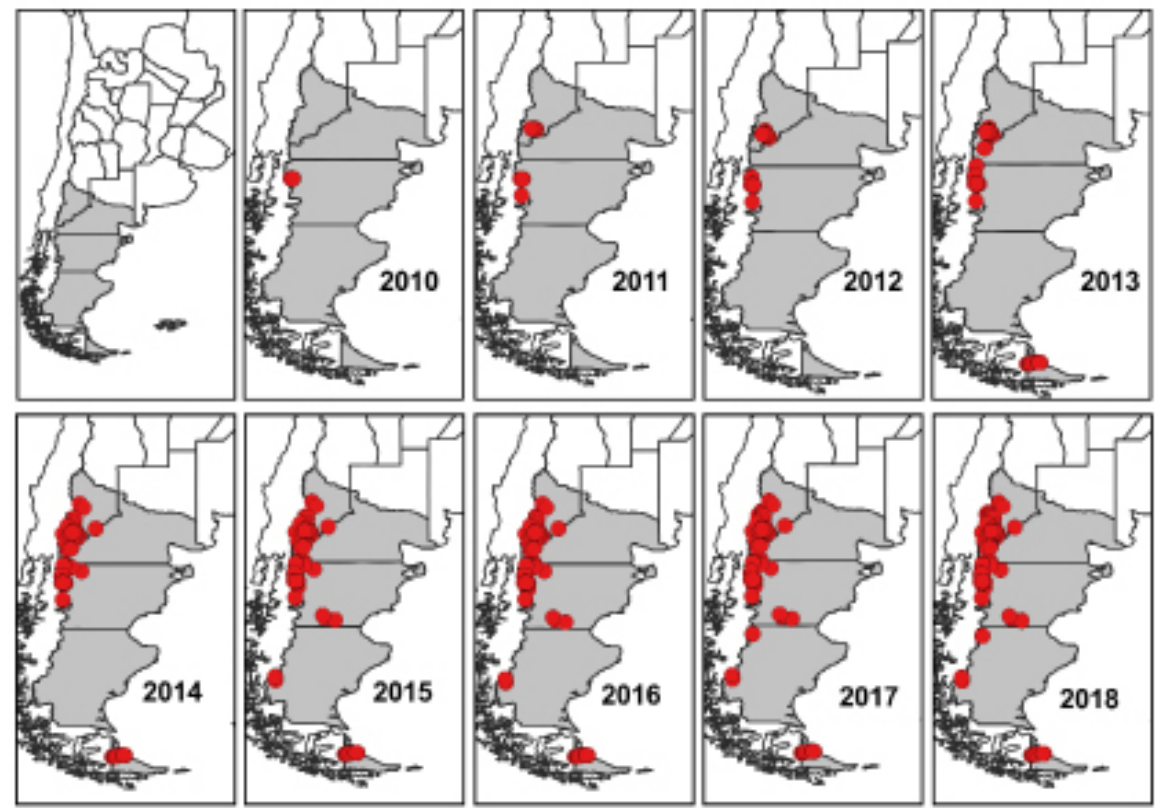

Fig. 6. Distribución acumulada año a año de D. geminata en toda la región patagónica (2010-2018). 


\section{Discusión}

Durante todo el período analizado, desde su primera aparición en 2010 hasta abril de 2018, pueden mencionarse nuevas apariciones, esto es registros positivos en sitios anteriormente muestreados con resultado negativo. Para el caso de la provincia del Chubut, que tiene el registro más completo y continuo, puede decirse que hubo un avance de $D$. geminata en el territorio provincial con punto de partida en el río Futaleufú en 2010, extendiéndose hacia el norte y el sur de la misma, y sobrepasando en 2014 los límites de la ecorregión Bosques Patagónicos, alcanzando la Estepa Patagónica.

Para el caso de gran parte de las provincias del Neuquén y Río Negro, si bien se cuenta con datos de 8 años, no es posible establecer un patrón geográfico dispersivo ya que no hay repetición de los sitios muestreados. Sólo en la zona suroeste del Neuquén, en el departamento de Aluminé, el grupo de trabajo ha muestreado semestralmente en sitios seleccionados con continuidad desde 2016 hasta 2018 observando un avance de la invasión a lo largo del río Aluminé y en los alrededores del lago del mismo nombre y del lago Moquehue. En las provincias de Santa Cruz y Tierra del Fuego, al no contar con información de varios años no es posible establecer patrones de distribución.

\section{Conclusiones}

En la actualidad la presencia de D. geminata está confirmada desde los $38^{\circ}$ a los $56^{\circ}$ Latitud Sur y desde los $73^{\circ}$ a los $67^{\circ}$ Longitud Oeste, lo que no significa que su distribución no pueda ser más amplia. Es importante considerar en este primer análisis general la discontinuidad de los muestreos en el espacio y en el tiempo. Solo en Chubut existen registros continuos en el tiempo y en menor medida en Neuquén. Sin embargo, el análisis de los sitios que fueron visitados en más de una oportunidad con resultados negativos y posteriormente positivos, evidencian la rápida expansión de la especie. Dado que no existe un cuerpo de información sobre parámetros ambientales, no es posible plantear hipótesis acerca de cuáles han sido los factores que favorecieron la expansión de la especie a nivel regional. La generación de este SIG constituye una herramienta de base y análisis que en esta primera instancia permite conocer la expansión de $D$. geminata en la Patagonia argentina desde su primera aparición en 2010. Esta base geográfica de datos será actualizada a medida que se vaya generando nueva información. En una etapa posterior serán realizados análisis espaciales más complejos, incluyendo la dinámica de nichos, que permitirán establecer la dinámica de la invasión actual y establecer sitios susceptibles de ser invadidos por la especie.

\section{CoNTRIBUCIÓN DE LOS AUTORES}

AL, JP y SS diseñaron la investigación, realizaron la base de datos y analizaron la información, UN, VS, NS y JMS planificaron y ejecutaron los muestreos en las provincias del Chubut y Neuquén y analizaron las muestras biológicas. Todos los autores participaron en la escritura del manuscrito.

\section{Agradecimientos}

Este trabajo se realizó con fondos del Proyecto N720 de la UNLP, Proyecto de Investigación SCyT UNPSJB, Res. R8/517/16 y Proyecto "Cuidemos nuestros ríos" No 1049 del Programa de Voluntariado Universitario del Ministerio de Educación de la Nación.

\section{Bibliografía}

ABELLI BONARDI, M., G. BAFFICO, G. BEAMUD, L. CASTIÑEIRA, M. DÍAZ, L. LAFFITTE, B. LAURENZANO, P. MORZENTI, J. MUÑIZ SAAVEDRA, F. PEDROZO, D. RIVERA \& P. TEMPORETTI. 2012. Programa de Vigilancia, seguimiento y control de Didymosphenia geminata (Lyngbye) Schmidt 1899 en la provincia del Neuquén. V Congreso Argentino de Limnología, 28 al 1 de diciembre de 2012. Instituto Nacional de Limnología, Santa Fé.

ASPREY, G. F., K. BENSON-EVANS \& J. FURET. 1964. A contribution to the study of South American freshwater phytoplankton. Gayana Bot. 10: 1-18. 


\section{A. A. Lamaro et al. - Didymosphenia geminata en Argentina}

BEAMUD, G., G. D. BAFFICO, F. PEDROZO \& M. DIAZ. 2013. First record of the invasive algae Didymosphenia geminata in the Lake Nahuel Huapi: Argentina, Patagonia. Rev. Chil. Hist. Nat. 86: 493496.

https://doi.org/10.4067/S0716-078X2013000400012

BEAMUD, S. G., G. BAFFICO, B. REID, R. TORRES, M. GONZALEZ-POLO, F. PEDROZO \& M. DIAZ. 2016. Photosynthetic performance associated with phosphorus availability in mats of Didymosphenia geminata (Bacillariophyceae) from Patagonia (Argentina and Chile). Phycologia 55: 118-125. https://dx.doi.org/10.2216/15-83.1

BEHRENDT, H. \& D. OPITZ. 2001. Preliminary approaches for the classification of rivers according to the indicator phytoplankton. In: BÄCK S. \& K. KARTTUNEN (eds.), Classification of ecological status of lakes and rivers, pp. 32-36. Nordic Council of Ministers, Copenhagen.

BLANCO, S. \& E. BECARES MANTECÓN. 2009. Una nueva alga invasora llega a España: Didymosphenia geminata. Quercus 283: 30-34.

BLANCO, S. \& L. ECTOR. 2009. Distribution, ecology and nuisance effects of the freshwater invasive diatom Didymosphenia geminata (Lyngbye) M. Schmidt: a literature review. Nova Hedwigia 88: 347-422.

https://doi.org/10.1127/0029-5035/2009/0088-0347

BOTHWELL, M. L. \& S. A. SPAULDING. 2008. Synopsis of the 2007 International Workshop on Didymosphenia geminata. In: BOTHWELL, M. L. \& S. A. SPAULDING (eds.), Proceedings of the 2007 International Workshop on Didymosphenia geminata. Can. Tech. Rep. Fish. Aquat. Sci. 2795, pp. 13-21.

BOTHWELL, M. L., B. W. TAYLOR \& C. KILROY. 2014. The Didymo story: the role of low dissolved phosphorus in the formation of Didymosphenia geminata blooms. Diatom Res. 29: 229-236.

https://doi.org/10.1080/0269249X.2014.889041

BRACCALENTI, J. 2016. Plan de Monitoreo de Didymo (Didymosphenia geminata) en cuerpos de agua de la provincia de Santa Cruz. Informe final. Julio 2016. Provincia de Santa Cruz. Consejo Federal de Inversiones. $78 \mathrm{pp}$.

BURROUGH, P. A. 1986. Principles of Geographic Information Systems for Land Resource Assessment. Clarendon Press, Oxford.

BUS LEONE, P., J. CERDA, S. SALA. \& B. REID. 2014. Mink (Neovison vison) as a natural vector in the dispersal of the diatom Didymosphenia geminata. Diatom Res. 29: 259-266.

https://doi.org/10.1080/0269249X.2014.890957

CASCO, M. A. \& S. E. SALA. 2012. Protocolo para

la detección y seguimiento de Didymosphenia geminata (Lyngbye) Schmidt en el área andina patagónica, Argentina. Autoridad Interjurisdiccional de las Cuencas de los Ríos Limay, Neuquén y Negro, Secretaría de Gestión Ambiental, Cipolletti. 57 pp.

CHAPMAN, V. J., R. H. THOMPSON \& E. C. M. SEGAR. 1957. Check list of the fresh-water algae of New Zealand. Trans. Roy. Soc. New Zealand 84: 695-747.

DAY, S. A., R. P. WICKHAM, T. J. ENTWISLE \& P. A. TYLER. 1995. Bibliographic check-list of nonmarine algae in Australia. Flora of Australia Supplementary Series 4: 1-276.

DUNCAN, M., C. KILROY, C. VIEGLAIS \& F. VELVIN. 2007. Protocol for the collection of samples for delimiting surveys for Didymosphenia geminata for microscopic analysis. National Institute of Water \& Atmospheric Research, New Zealand. Client Report: CHC2007-110, 19 pp.

FITZHUGH, T. W. 2005. GIS Tools for Freshwater Biodiversity Conservation Planning. Trans. GIS 9: 247-263.

https://doi.org/10.1111/j.1467-9671.2005.00215.x

GIANSANTE, C. \& S. PELINI. 2007. The use of geographic information systems in sea and freshwater ecosystems. Vet. Ital. 43: 507-512.

GILliS, C. A. \& M. CHALIFOUR. 2010. Changes in the macrobenthic community structure following the introduction of the invasive algae Didymosphenia geminata in the Matapedia River (Québec, Canada). Hydrobiologia 647: 63-70.

https://doi.org/10.1007/s10750-009-9832-7

HASLETT, J. R. 1990. Geographic information systems. A new approach to habitat definition and study of distributions. Trends Ecol. Evol. 5: 214-218. https://doi.org/10.1016/0169-5347(90)90134-Y

HIJMANS, R. J., M. CRUZ, E. ROJAS \& L. GUARINO. 2001. DIVA-GIS version 1.4: A geographic information system for the analysis of biodiversity data. Manual. International Potato Center, Lima, Perú.

JAMES, D. A. \& S. R. CHIPPS. 2016. Influence of Didymosphenia geminata blooms on prey composition and associated diet and growth of Brown Trout. Trans. Am. Fish. Soc 145: 195-205. https://doi.org/10.1080/00028487.2015.1111255 
JONES, P., S. BEEBE, J. TOHME \& N. GALWEY. 1997. The use of geographical information systems in biodiversity exploration and conservation. Biodivers. Conserv. 6: 947-958. https://doi.org/10.1023/A:1018311513176

KILROY, C. 2004. A new alien diatom Didymosphenia geminata (Lyngbye) Schmidt: its biology, distribution, effects and potential risks for New Zealand fresh waters. NIWA Client Report CHC2004-128.

KILROY, C. 2005. Tests to determine the effectiveness of methods for decontaminating materials that have been in contact with Didymosphenia geminata. NIWA Client Report: CHC2005-005, Project MAF05501.

KILROY, C. 2008. Didymosphenia geminata in New Zealand: Distribution, dispersion and ecology of a non-indigenous invasive species. In: BOTHWELL, M. L. \& S. A. SPAULDING (eds.), Proceedings of the 2007 International Workshop on Didymosphenia geminata. Can. Tech. Rep. Fish. Aquat. Sci. 2795, pp. $15-20$.

KILROY, C. \& M. DALE. 2006. A comparison of sampling methods for the detection of the invasive alga Didymosphenia geminata in New Zealand. NIWA Client Report: CHC2006-078.

KILROY, C. \& P. NOVIS. 2018. Is Didymosphenia geminata an introduced species in New Zealand? Evidence from trends in water chemistry, and chloroplast DNA. Ecol. Evol. 8: 904-919. https://doi.org/10.1002/ece3.3572

KILROY, C. \& M. UNWIN. 2011. The arrival and spread of the bloom-forming, freshwater diatom, Didymosphenia geminata, in New Zealand. Aquat. Invasions 6: 249-262. https://doi.org/10.3391/ai.2011.6.3.02

KILROY, C., T. SNELDER \& J. SYKES. 2005. Likely environments in which the nonindigenous freshwater diatom can survive, in New Zealand. NIWA Consultancy Report 2005-043.

KILROY, C., A. LAGERSTEDT, A. DAVEY \& K. ROBINSON. 2007. Studies on the survivability of the exotic, invasive diatom Didymosphenia geminata under a range of environmental and chemical conditions. NIWA Report: CHC2006116.

KUMAR, S., S. A. SPAULDING, T. J. STOHLGREN, K. A. HERMANN, T. S. SCHMIDT \& L. L. BAHLS. 2009. Potential habitat distribution for the freshwater diatom Didymosphenia geminata in the continental US. Front. Ecol. Environ. 7: 415-420. https://doi.org/10.1890/080054
LARNED S, B. BIGGS, N. BLAIR, C. BURNS, B. JARVIE, D. JELLYMAN, C. KILROY, J. LEATHWICK, K. LISTER, J. NAGELS, M. SCHALLENBERG, S. SUTHERLAND, J. SYKES, W. THOMPSON, K. VOPEL \& B. WILCOCK. 2007. Ecological studies of Didymosphenia geminata in New Zealand, 2006-2007. NIWA. New Zealand. Client Report CHC2007-070, NIWA Project MAF07507.

LiNDSTRØM, E. \& O. M. SKULBERG. 2008. Didymosphenia geminata - a native diatom species of Norwegian rivers coexisting with the Atlantic salmon. In: BOTHWELL, M. L. \& S. A. SPAULDING (eds.). Proceedings of the 2007 International Workshop on Didymosphenia geminata, Can. Tech. Rep. Fish. Aquat. Sci. 2795, pp. 35-40.

MCNYSET, K. M. \& M. L. JULIUS. 2006. Potential geographic distribution of Didymosphenia geminata (Lyngbye) M. Schmidt in North America based on ecological niche models. Abstract. Region 8 Biomonitoring and Bioassessment Meeting - April 4-6, 2006 - Utah State University, Logan.

MOREIRA-MUÑOZ, A. 1996. Los Sistemas de Información Geográfica (SIG) y sus aplicaciones en la conservación de la diversidad biológica. Amb. Des. 12: $80-86$.

MORENO, P., L. MOLINARI, J. KURODA, M. DEMICHELI \& P. HUALDE. 2011. Primer reporte del alga invasora Didymosphenia geminata en el río Collón Cura, Provincia del Neuquén. Informe $\mathrm{N}^{\circ} 9$. Departamento de Fauna Acuática Centro de Ecología Aplicada del Neuquén, Ministerio de Desarrollo Territorial Provincia del Neuquén. 6 pp.

NAUMOSKI, A., G. MIRCEVA. \& K. MITRESKI. 2011. Fuzzy models with GIS for water quality diatom-indicator classification, In: 2011 Eighth International Conference on Fuzzy Systems and Knowledge Discovery (FSKD). IEEE, pp. 829-833. https://doi.org/10.1109/FSKD.2011.6019732

POTAPOVA, M. G. \& D. F. CHARLES. 2002. Benthic diatoms in USA rivers: distributions along spatial and environmental gradients. J. Biogeogr. 29: 167-187. https://doi.org/10.1046/j.1365-2699.2002.00668.x

RAMACHANDRA, T. V. \& N. AHALYA. 2001. Monograph on Essentials in Limnology and GIS. Karnataka Environment Research Foundation, Bangalore.

REID, B. L., K. L. HERNANDEZ, M. FRANGOPULOS, G. BAUER, M. LORCA, C. KILROY \& S. SPAULDING. 2012. The invasion of the freshwater 


\section{A. A. Lamaro et al. - Didymosphenia geminata en Argentina}

diatom Didymosphenia geminata in Patagonia: prospects, strategies, and implications for biosecurity of invasive microorganisms in continental waters. Conserv. Lett. 5: 432-440. https://doi.org/10.1111/j.1755-263X.2012.00264.X

REVENGA, C., I. CAMPBELL, R. ABELL, P. DE VILLIERS \& M. BRYER. 2005. Prospects for monitoring freshwater ecosystems towards the 2010 targets. Philos. Trans. Roy. Soc. B Biol. Sc. 360: 397-413.

RIVERA, P. \& M. GEBAUER. 1989. Diatomeas chilenas en las Colecciones de Boyer, Cleve \& Moeller, Schulze y Smith, depositadas en la Academia de Ciencias Naturales de Filadelfia, Estados Unidos. Gayana Bot. 46: 89-116.

SALA, S. E., S. SPAULDING, M. E. FERRARIO \& A. A. LAMARO. 2013. Presencia de Didymosphenia geminata en Tierra del Fuego. In: XXXIV Jornadas Argentinas de Botánica. Bol. Soc. Argent. Bot. 48 (Supl.): 174-175.

SALEM, B. B. 2003. Application of GIS to biodiversity monitoring. J. Arid Environ. 54: 91-114. https://doi.org/10.1006/jare.2001.0887

SANMIGUEL, A., S. BLANCO, I. ALVAREZ BLANCO, C. CEJUDO FIGUEIRAS, A. ESCUDERO, M. E. PEREZ, G. NOYON \& E. BECARES. 2016. Recovery of the algae and macroinvertebrate benthic community after Didymosphenia geminata mass growths in Spanish rivers. Biol. Invasions 18: $1467-$ 1484. https://doi.org/10.1007/s10530-016-1095-4

SASTRE, V., G. BAUER \& M. G. AYESTARÁN. 2010a. Monitoreo de Didymosphenia geminata. Informe $\mathrm{N}^{\mathrm{o}}$ 5: Resultados Muestreo 31 de Agosto, 2 y 7 de Septiembre de 2010. Laboratorio de Hidrobiología, Facultad de Ciencias Naturales, Sede Trelew-Esquel.

SASTRE, V., G. BAUER \& M. G. AYESTARÁN. 2010 b. Monitoreo de Didymosphenia geminata. Informe $\mathrm{N}^{\mathrm{o}}$ 8: Resultados Muestreo 21 de Septiembre de 2010. Laboratorio de Hidrobiología, Facultad de Ciencias Naturales, Sede Trelew-Esquel. 12 pp.
SASTRE, A. V., N. H. SANTINELLI, G. A. BAUER, M. G. AYESTARAN \& N. M. UYUA. 2013. First record of the invasive diatom Didymosphenia geminata (Lyngbye) Schmidt in a Patagonian Andean river of Argentina. Bioinvasions Rec. 2: 1117. http://dx.doi.org/10.3391/bir.2013.2.1.02

SHULTZ, L. 2000. Using geographical information systems in floristic studies. SIDA, Bot. Misc. 18: 73-81.

SPAULDING, S. A. \& L. ELWELL. 2007. Increase in nuisance blooms and geographic expansion of the freshwater diatom Didymosphenia geminata: U.S. Geological Survey Open-File Report 2007-1425. 38 pp. https://doi.org/10.3133/ofr20071425

TAYLOR, B. W. \& M. L. BOTHWELL. 2014. The origin of invasive microorganisms matters for science, policy, and management: The case of Didymosphenia geminata. Bioscience 64: 531-538. https://doi.org/10.1093/biosci/biu060

UYUA, N. M., N. H. SANTINELLI, A. V. SASTRE \& S. E. SALA. 2016. Morfología valvar de Didymosphenia geminata (Bacillariophyceae) en la provincia de Chubut, Argentina. Bol. Soc. Argent. Bot. 51: 15-27. https://doi.org/10.31055/1851.2372.v51.n1.14366

UYUA, N. M. 2017. Didymosphenia geminata (Lyngbye) M. Schmidt en el área andina de la provincia de Chubut: taxonomía, diversidad morfológica y genética y origen del alga invasora. Tesis Doctoral $\mathrm{N}^{\circ}$ 1494. Facultad de Ciencias Naturales y Museo, UNLP.

VOUILloud, A. A. 2003. Catálogo de diatomeas continentales y marinas de Argentina. Asociación Argentina de Ficología, La Plata. Versión 1.0 (Formato digital).

Whitton, B. A., N. T. W. ELlWOOD \& B. KAWECKA. 2009. Biology of the freshwater diatom Didymosphenia: a review. Hydrobiologia 630: $1-37$.

https://doi.org/10.1007/s10750-009-9753-5 
